\title{
Directed Orientation of Asymmetric Composite Dumbbells by Electric Field Induced Assembly
}

\author{
Daisuke Nagao, ${ }^{\dagger}$ Maki Sugimoto, ${ }^{\dagger}$ Ayako Okada, ${ }^{\dagger}$ Haruyuki Ishii, ${ }^{\dagger}$ Mikio Konno, ${ }^{*}{ }^{\dagger}$ Arnout Imhof, ${ }^{\ddagger}$ \\ and Alfons van Blaaderen ${ }^{*}+$
} ${ }^{\dagger}$ Department of Chemical Engineering, Tohoku University, 6-6-07 Aoba, Aramaki-aza Aoba-ku, Sendai, 980-8579, Japan
${ }^{\ddagger}$ Soft Condensed Matter Group, Debye Institute for Nanomaterials Science, Utrecht University, Princetonplein 5, 3584 CC Utrecht,
The Netherlands

\section{Supporting Information}

ABSTRACT: Assembly and directed orientation of anisotropic particles with an external ac electric field in a range from $1 \mathrm{kHz}$ to $2 \mathrm{MHz}$ were studied for asymmetric composite dumbbells incorporating a silica, titania, or titania/silica (titania:silica $=75: 25$ vol \%) sphere. The asymmetric composite dumbbells, which were composed of a polymethylmethacrylate (PMMA)-coated sphere (core-shell part) and a polystyrene (PSt) lobe, were synthesized with a soap-free emulsion

Electric Field Induced Assembly Controllable Particle Orientation Highly dielectric material (Titania sphere)

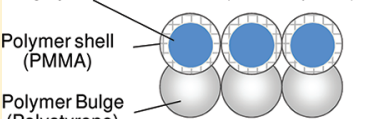
Polymer Bulge
(Polystyrene)

Perpendicular to the field at low frequency $(\sim \mathrm{kHz})$

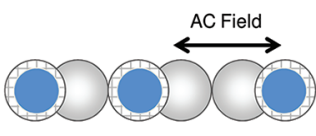

Parallel to the field at high frequency $(\sim \mathrm{MHz})$ polymerization to prepare PMMA-coated inorganic spheres and another soap-free emulsion polymerization to form a polystyrene (PSt) lobe from the PMMA-coated inorganic spheres. The composite dumbbells dispersed in water were directly observed with optical microscopy. The dumbbells incorporating a silica sphere oriented parallel to an electric field in the whole frequency range and they formed a pearl chain structure at a high frequency of 2 $\mathrm{MHz}$. The titania-incorporated dumbbells formed chain structures, in which they contacted their core-shell parts and oriented perpendicularly to a low-frequency $(\mathrm{kHz})$ field, whereas they oriented parallel to a high-frequency $(\mathrm{MHz})$ field. Since the alignment of dumbbells in the chains depends not only on the interparticle forces but also on the torque that the induced dipoles in the dumbbells experience in the electric field, the orientation of dumbbells perpendicular to the electric field was the case dominated by the interparticle force, whereas the other orientation was the case dominated by the torque. The present experiments show that the incorporation of inorganic dumbbells is an effective way to control the assembled structure and orientation with an electric field.

\section{INTRODUCTION}

The importance of creating new types of anisotropic particles has recently been emphasized, because a number of researchers have shown that anisotropic particles have potential for exhibiting unique properties in various fields, such as optics, ${ }^{1,2}$ catalysis, ${ }^{3}$ and suspension rheology. ${ }^{4}$ The unique properties can be diversified by controlling positional and orientational order of the anisotropic particles in an assembly. Liddell et al. employed a convective assembly method and succeeded in fabricating $2 \mathrm{D}$ ordered arrays of asymmetric polymer dumbbells oriented in-plane or predominantly out-of-plane. ${ }^{5}$ It was suggested in their report that orientation of the asymmetric dumbbells depended on the concentration of dumbbells through packing constraints in the region of a receding meniscus.

External magnetic fields have also been examined to orient anisotropic colloids in the process of self-assembly. ${ }^{6,7}$ The above-mentioned convective assembly method, this time supplemented by the use of an externally applied magnetic field, was used to fabricate 3D photonic crystals composed of magnetic ellipsoids. ${ }^{8}$ Novel types of colloidal clusters with chiral" and "crisscross" structures fabricated from magnetic colloids with shapes resembling peanuts ${ }^{10}$ were created by the application of magnetic fields to anisotropic colloids.
Another effective way to orient anisotropic particles is to apply electric fields to suspensions of anisotropic particles. Monodisperse, anisotropic particles with disk, rod, and fenestrated hexagon shapes ${ }^{11}$ were studied with fluorescence microscopy to observe the orientation of the anisotropic particles under an ac electric field. The anisotropic particles were observed to form chains in which they preferentially oriented with their longest axes parallel to the electric field. Similar orientations of anisotropic particles under applied electric fields were observed for polystyrene ellipsoids in chainlike structures ${ }^{12}$ and also for snowman-shaped poly(methyl methacrylate) particles coated with a conducting polymer of polyaniline in silicone oil as an electrorheological (ER) fluid. ${ }^{4}$ Silica dumbbells with the shape of diatomic molecules were observed under an electric field with confocal microscopy revealing several new crystal structures. A martensitic crystal-crystal transition was found for the silica dumbbells by varying the electric field strength to promote dipole-dipole interactions. ${ }^{13}$ Also micrometer-sized rodlike silica particles were observed to orient with their long axis in

Received: November 15, 2011

Revised: $\quad$ March 29, 2012

Published: March 29, 2012 
the direction of the electric field in preliminary measurements on smectic phases of such particles. ${ }^{14}$ On the other hand, it was also reported that some anisotropic particles, such as rodlike fd virus particles ${ }^{15}$ and ellipsoidal titania particles, ${ }^{16}$ could be oriented perpendicularly to applied electric fields and exhibited electric birefringence of the particle assemblies. However, the orientation of anisotropic particles parallel or perpendicular to electric fields has not been studied experimentally in detail yet, and also the number of simulation studies focusing on electric field effects of phase behavior of polarizable anisotropic particles under electric fields is very limited. ${ }^{17}$

To date, homogeneous organic or inorganic particles have been used in the self-assembly of anisotropic particles under electric fields. However, the use of composite particles of organic and inorganic components is a promising method, because it would allow induced dipolar interactions between distinct parts of the anisotropic particles to be optimized for (switchable) control over the orientation, long-range order, and packing.

We have recently developed a method to synthesize monodisperse, composite dumbbells incorporating an asymmetrically placed inorganic sphere. ${ }^{18,19}$ The synthetic method consists of a soap-free emulsion polymerization to prepare PMMA-coated inorganic spheres and another soap-free emulsion polymerization to form a polystyrene (PSt) lobe from the PMMA-coated inorganic spheres (see synthetic scheme in Figure S1, Supporting Information). A characteristic feature of the asymmetric dumbbells is a combination of polymeric and inorganic spheres joined to each other in a dumbbell, which can be used to control the relative polarization of the dumbbell lobes under applied electric fields. By using this characteristic feature, we here propose a method to gain control over the positional and orientational order of the asymmetric composite dumbbells. In our method, an ac electric field in a wide frequency range from kilohertz to megahertz is applied to explore the strong interactions between inorganic substances incorporated into the dumbbells. It was reported that titania has a dielectric constant $\left(47\right.$ at $1 \mathrm{kHz}^{22}$ and 5.5 at $1 \mathrm{MHz}^{23}$ ) higher than that of silica $\left(3.8\right.$ at $1 \mathrm{kHz}^{24}$ and $\sim 2.0$ at $\left.1 \mathrm{MHz}^{25}\right)$. Three different asymmetric dumbbells, incorporating a silica, titania, or titania/silica (titania:silica $=75: 25$ vol \%) sphere, are employed to examine the interactions between the inorganic parts of dumbbells under the electric field.

\section{EXPERIMENTAL SECTION}

Chemicals. Titanium tetraisopropoxide (TTIP, 95\%), ethanol (99.5\%), acetonitrile (99.5\%), methylamine aqueous solution (40\%), methyl methacrylate (MMA, 98\%), styrene (St, 99\%), sodium pstyrenesulfonate (NaSS), potassium persulfate (KPS, 95\%), and sodium chloride $(\mathrm{NaCl}, 99.5 \%)$ were perchased from Wako Pure Chemical Industries (Osaka, Japan). The inhibitors for monomers of MMA and St were removed by an inhibitor removal column. The other chemicals were used as received. The silane coupling agent 3methacryloxypropyltrimethoxysilane (MPTMS, 95\%) was obtained from Shinetsu Chemical (Tokyo, Japan) and used as received.

Particle Synthesis. Sol-gel reaction and soap-free emulsion polymerization were applied to prepare asymmetric polymer dumbbells with an inorganic core of titania, silica, and titania/silica. ${ }^{18}$

The titania core particles were prepared via hydrolysis and condensation of TTIP conducted for $12 \mathrm{~h}$ in a mixed solvent of ethanol and acetonitrile in a glass flask reactor $(400 \mathrm{~mL}$ vol) with initial concentrations of $0.03 \mathrm{M}$ TTIP, $0.1 \mathrm{M}$ water, and $0.03 \mathrm{M}$ methylamine at $10{ }^{\circ} \mathrm{C} .{ }^{20}$ Resultant particles were amorphous titania and were washed by centrifugation and redispersion in deionized water. Then, two-step polymerizations of MMA and St were conducted in the presence of the titania particles. In the MMA polymerization, deionized water $(192 \mathrm{~g})$ was bubbled with nitrogen for $30 \mathrm{~min}$, followed by the addition of MPTMS $(114 \mu \mathrm{L})$ and a $4.57 \mathrm{wt}$ $\%$ aqueous titania particle suspension $(9.1 \mathrm{~g})$ to the water. After 30 min of stirring, MMA $(5.1 \mathrm{~mL})$ and a $20 \mathrm{~mL}$ aqueous solution containing $0.0375 \mathrm{~g}$ of NaSS were added to the titania suspension. After $2 \mathrm{~h}$ of stirring at $35^{\circ} \mathrm{C}$, the mixed solution was heated to $65{ }^{\circ} \mathrm{C}$ and a $20 \mathrm{~mL}$ aqueous solution containing KPS $(0.130 \mathrm{~g})$ was added to initiate polymerization. The polymerization was performed at $300 \mathrm{rpm}$ for $2 \mathrm{~h}$ at $65{ }^{\circ} \mathrm{C}$. The PMMA-coated titania particles obtained were washed three times and redispersed into $30 \mathrm{~mL}$ of deionized water. In the St polymerization, the suspension of the PMMA-coated particles $(7.17 \mathrm{~g})$ and $0.1 \mathrm{M} \mathrm{NaCl}$ solutions $(5.6 \mathrm{~g})$ were added to $17.52 \mathrm{~g}$ of deionized water. After $30 \mathrm{~min}$ of bubbling, $0.921 \mathrm{~mL}$ of St was added to the suspension and the mixture was stirred for $2 \mathrm{~h}$ at room temperature. Finally, the mixture was heated to $65^{\circ} \mathrm{C}$ and a $10 \mathrm{~mL}$ aqueous solution containing KPS $(0.0219 \mathrm{~g})$ was added. The resultant suspension was washed by centrifugation and redispersion in deionized water.

The composite dumbbells with a silica or titania/silica core were synthesized by a similar procedure via sol-gel reaction and double polymerizations of MMA and St. Details of the procedure are described in the Supporting Information. With the present synthetic procedure, almost the same shape and size of asymmetric dumbbells were produced for the three different cores of silica, titania, and titania/silica. The synthetic conditions for the inorganic spheres are listed in Table S1 of the Supporting Information.

Setup for Application of Electric Fields. ${ }^{21}$ The sample cell used for particle observation under electric fields consisted of a capillary $(0.1 \times 1 \mathrm{~mm}$ rectangular cross section, VitroCom) and two $50 \mu \mathrm{m}$ diameter copper wires (99.99\%, NIRACO) threaded through along the side walls. The capillary was filled with an aqueous suspension of particles, and the ends of capillary were sealed with glue. The ac field (up to $V_{\text {rms }}=70.7 \mathrm{~V} / \mathrm{mm}$ ) was applied by connecting the copper wires to a function generator (GWINTEK, SFG-2004) and amplifier (NF Circuit Design Bloc, HSA4011). The electric field strength (peak to peak) was measured with a digital oscilloscope (GWINTEK, GDS$1062 \mathrm{~A}$ ). The weight fraction of inorganic spheres in the suspensions was $0.1 \mathrm{wt} \%$ to acquire clear images in observation with an optical microscope $(\mathrm{OM})$. All OM images were taken at $30 \mathrm{~s}$ after initiation of applying electric fields.

Characterization. The resulting particles were observed with a transmission electron microscope (TEM, Carl Zeiss, LEO912AB), a scanning transmission electron microscope (STEM, Hitachi, HD2700), and an optical microscope (OLYMPUS, BH-2). In preparation of samples for TEM and STEM, a small amount of resultant solution was introduced onto a microgrid and dried at room temperature.

\section{RESULTS AND DISCUSSION}

Figure 1 shows TEM images of the asymmetric composite dumbbells prepared with the previously proposed method. ${ }^{18}$ The TEM images, in which inorganic spheres are shown as black circles, clearly indicate that one part of the dumbbells contains an inorganic sphere. Characteristic dimensions of the dumbbells shown in Figure1 were measured in terms of length of the long axis of the dumbbell $(L)$, PMMA-coated sphere size $(S)$, polystyrene lobe size $(P)$, and diameter of the dumbbell neck $(D)$. Their average values and coefficients of variations $\left(C_{\mathrm{V}}\right)$ are listed in the Supporting Information (Table S2). The dumbbells with the three different cores all had an average long axis $\left(L_{\text {ave }}\right)$ in the range of $1.8-2.0 \mu \mathrm{m}$, an average size ratio $\left(P_{\text {ave }} / S_{\text {ave }}\right)$ of $0.91-0.92$, and an average aspect ratio $\left[L^{*}=\right.$ $\left.2 L_{\text {ave }} /\left(P_{\text {ave }}+S_{\text {ave }}\right)\right]$ of $1.7-1.8$. The dumbbells were welldispersed in water and their Brownian motion could be clearly observed with an optical microscope.

Electric fields with $V_{\text {rms }}=70.7 \mathrm{~V} / \mathrm{mm}$ at frequencies of $1 \mathrm{kHz}$ and 1 and $2 \mathrm{MHz}$ were applied to suspensions of the three 


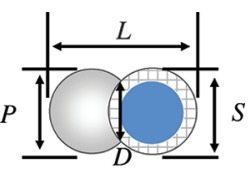

(A) $\mathrm{SiO}_{2}$ spheres

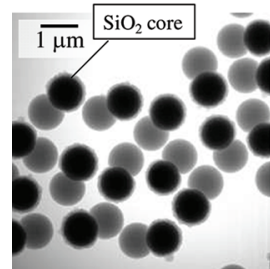

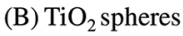

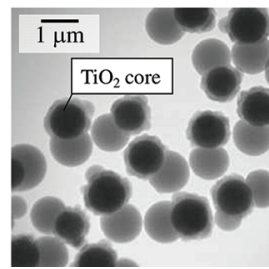

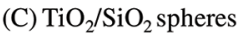

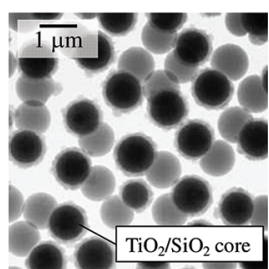

Figure 1. TEM images of asymmetric composite dumbbells incorporating a silica sphere (A), a titania sphere (B), and a $\mathrm{TiO}_{2} /$ $\mathrm{SiO}_{2}$ sphere $\left(\mathrm{C}, \mathrm{TiO}_{2}: \mathrm{SiO}_{2}=75: 25\right.$ vol \%).

asymmetric dumbbells shown in Figure 1. Of these frequencies, the electric field at $1 \mathrm{kHz}$ induced strong electro-osmotic flows that disturbed the observation of our asymmetric dumbbells with an optical microscope (OM). On the other hand, the electric fields at 1 and $2 \mathrm{MHz}$ did not cause electro-osmotic flows, because at these high frequencies the double layer ions are not able to follow the changes in field directions. For the following observations of dumbbells, the field strength of 17.7 $\mathrm{V} / \mathrm{mm}$ was applied to suppress the electro-osmotic effect at 1 $\mathrm{kHz}$ whereas $70.7 \mathrm{~V} / \mathrm{mm}$ was employed at 1 and $2 \mathrm{MHz}$.

Figure 2 shows OM images of the asymmetric polymer dumbbells incorporating a silica sphere observed under electric

Frequency
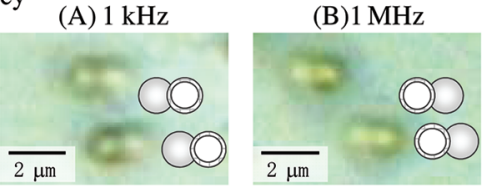

$2 \mu \mathrm{m}$

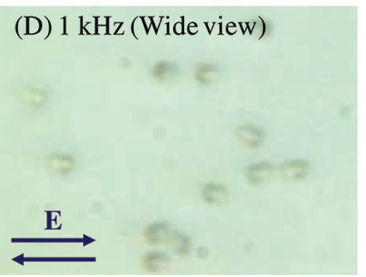

(E) $2 \mathrm{MHz}$ (Wide view)

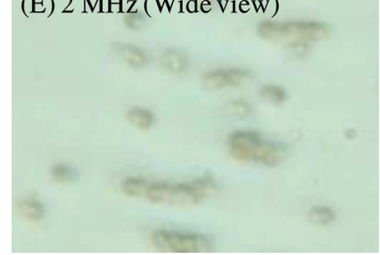

Figure 2. Optical microscope images of asymmetric dumbbells incorporating a silica sphere under electric fields at frequencies of 1 $\mathrm{kHz}(\mathrm{A}, \mathrm{D}), 1 \mathrm{MHz}(\mathrm{B})$, and $2 \mathrm{MHz}(\mathrm{C}, \mathrm{E})$. The strengths of electric fields were $V_{\mathrm{rms}}=17.7 \mathrm{~V} / \mathrm{mm}$ for $1 \mathrm{kHz}$ and $70.7 \mathrm{~V} / \mathrm{mm}$ for 1 and 2 $\mathrm{MHz}$.

fields at the three frequencies. The location of the silica spheres in the asymmetric dumbbells could be identified with the OM images due to difference in the refractive indices. These locations are illustrated in each $\mathrm{OM}$ image of Figure 2. The long axes of the dumbbells at $1 \mathrm{kHz}$ were orientated parallel to the electric field of $V_{\mathrm{rms}}=17.7 \mathrm{~V} / \mathrm{mm}$ (Figure 2A), but the dumbbells showed no preference for pointing left or right (see the wide view image of Figure 2D). A similar tendency of particle orientation was observed under a $1 \mathrm{MHz}$ electric field with $V_{\text {rms }}=70.7 \mathrm{~V} / \mathrm{mm}$ (Figure $2 \mathrm{~B}$ ). The high frequency of 2 $\mathrm{MHz}$ induced short chains parallel to the electric field, with particles randomly pointing left or right (see Figure 2C and the wide view of $\mathrm{E}$ ). Interactions due to electric double layers can be a factor in the orientation of asymmetric dumbbells in the low frequency range around $1 \mathrm{kHz}$, but this contribution to the interactions is generally much weaker at frequencies above 1 $\mathrm{MHz}$. Therefore, in the high frequency range, the electric double layer interaction can be ignored and only interactions resulting from dielectric polarization are the main factor determining particle orientation and chain formation.

For comparison, the electric field response of each component of the asymmetric dumbbells separately are shown as OM images in Figure S2 of the Supporting Information, where both silica spheres and PMMA-coated silica spheres are seen to readily form chains under the same electric fields. However, PSt particles scarcely formed the structures. The OM images indicate that the inorganic spheres incorporated into the asymmetric dumbbells enhance the formation of chain structures.

To clarify the effects of the inorganic component on the orientation of asymmetric dumbbells in their assembly, the same electric fields were applied to the other dumbbells incorporating amorphous titania or amorphous titania/silica sphere. Figure 3 shows $\mathrm{OM}$ images of these asymmetric

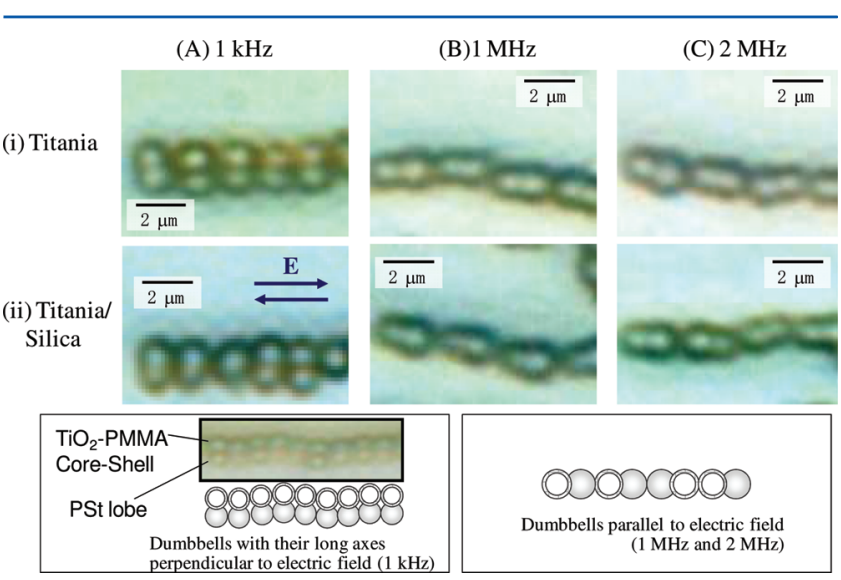

Figure 3. Optical microscope images of asymmetric dumbbells incorporating a titania (i) or titania/silica sphere (ii) under electric fields in a frequency range from $1 \mathrm{kHz}$ to $2 \mathrm{MHz}$. See Figure 2 for the strengths of the electric fields.

dumbbells. At frequencies of $1 \mathrm{MHz}$ or higher, the asymmetric dumbbells formed distinct chain structures parallel to the electric field with the long axes of the dumbbells parallel to the field. Comparison of these results at $1 \mathrm{MHz}$ with the corresponding result of the silica-core dumbbells in Figure.2B suggests that stronger interactions were induced between the dumbbells in Figure 3. Chain structures were also formed at 1 $\mathrm{kHz}$ in Figure 3, but interestingly, the dumbbells this time aligned with their long axes perpendicular to the electric field (also see Figure S3 of the Supporting Information for wide views). Although the dumbbells individually dispersed were aligned parallel to the electric field, the dumbbells forming chain structures were aligned with their long axes perpendicular to the electric field. Since the silica-core dumbbells did not form chains at all at the corresponding conditions in Figure 2, the results at $1 \mathrm{kHz}$ of Figure 3 shows that the incorporation of titania into the dumbbells increases the interactions between the dumbbells. This is supported by the observation of quicker and longer chain formation for titania spheres than that for silica ones at $1 \mathrm{kHz}$ (See movies M1 and M2, Supporting Information). The alignment of dumbbells in the chains 
depended not only on the interparticle forces but also on the torque on the effective dipoles induced by the electric field. It is likely at these lower frequencies that this torque was partially caused by effects in the double layer as well. It can be concluded that the long axes perpendicular to the dumbbell chain was the case dominated by the interparticle forces, while the other was the case dominated by the torque.

To examine the effect of particle permittivity on the chain structure, crystallized titania particles were also prepared, because they have a higher low-frequency permittivity than amorphous titania. These particles were made from the amorphous titania spheres by hydrothermal treatment at 150 ${ }^{\circ} \mathrm{C}$ for $3 \mathrm{~h}$ (see Figure S4, Supporting Information), followed by silica-coating, PMMA-coating, and finally PSt-protrusion formation. Figure 4 shows OM images comparing the behavior

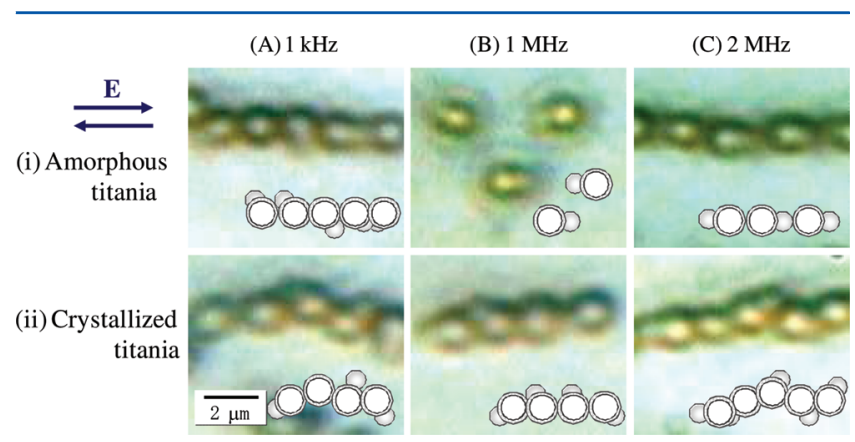

Figure 4. Optical microscope images of titania/silica core-shell particles with a small PSt lobe. The cores incorporated into the particles were (i) amorphous titania and (ii) crystallized titania. See Figure 2 for the strengths of the electric fields.

in electric fields of particles incorporating amorphous or crystallized titania. Both core-shell particles, with a smaller PSt lobe than before (snowman-shaped particles), had a long axis length $L$ of $1.6-1.8 \mu \mathrm{m}$, an aspect ratio $L^{*}$ of $1.67-1.77$, and a size ratio $P / S$ of $0.63-0.65$. The snowman-shaped particles incorporating amorphous titania (Figure 4i) contacted their core-shell parts at $1 \mathrm{kHz}$ without further particle orientation. Compared to Figure 3 the interaction between the smaller PSt lobes was now insufficient to cause orientation of the dumbbells. The chains dispersed again at $1 \mathrm{MHz}$, but with the particle orientation still parallel to the electric field, whereas they re-formed at $2 \mathrm{MHz}$. At this moment we do not have an explanation for this difference. It might be related to polarizations inside the shell as ions inside the colloids do not need to travel large distances to induce effects. On the other hand, the snowman-shaped particles incorporating crystalline titania preferentially contacted their titania-containing sides without dumbbell orientation throughout the whole frequency range. The preference for contacting the parts containing the crystallized titania spheres demonstrates the importance of core-particle permittivity to gain control over positional and orientational ordering of asymmetric dumbbells in Figure 3.

To examine the effects of the PSt lobe size on chain structure of asymmetric dumbbells, slightly PSt-protruded core-shell composite particles were observed with OM. Figure 5 shows OM images of the composite particles incorporating a silica or titania sphere under the electric fields. The silica and titania were both amorphous. The composite particles in Figure 5 have a long axis of $1.6 \mu \mathrm{m}$, an aspect ratio $L^{*}$ of 1.7 , and a size ratio $P / S$ of 0.74 . The composite particles incorporating silica were

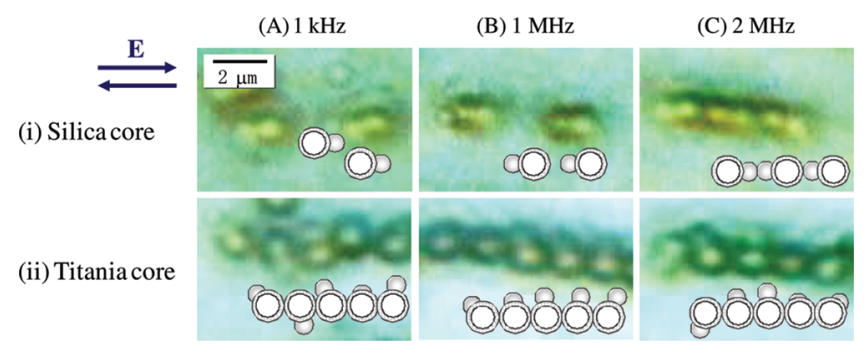

Figure 5. Optical microscope images of core-shell particles with a small PSt lobe. The cores incorporated into the particles were (i) silica and (ii) amorphous titania. See Figure 2 for the strengths of electric fields.

oriented parallel to the field in the whole frequency range but only formed chains at $2 \mathrm{MHz}$, similarly to the torquedominated cases observed in Figure 2. On the other hand, the composite particles incorporating titania contacted their core-shell parts without orientation in the whole frequency range, which was different from the assembly of titaniaincorporated dumbbells shown in Figure 3. Because of the smaller value of $L$, the torques applied to the dumbbells by the electric field in Figure 5 were smaller than in Figure 3. Therefore, the interparticle-force dominated chain formation, favoring a large number of contacts between the titania core lobes over dumbbell alignment.

\section{CONCLUSION}

Monodisperse, asymmetric composite dumbbells incorporating different inorganic cores were successfully prepared using double soap-free emulsion polymerizations. Asymmetric dumbbells incorporating a titania core (or a titania/silica core) formed chain structures in which they oriented perpendicularly to a low-frequency electric field and parallelly to a highfrequency field. Comparing this with chain structures observed for silica-incorporated dumbbells and snowman-shaped particles shows that introduction of highly dielectric materials into asymmetric particles allows the assembly and orientation of asymmetric particles into chains to be controlled with an electric field. Theoretical work and/or extensive simulation of the complex frequency dependent polarization behavior of these complex particles needs to be performed to fully understand this intriguing behavior that no doubt can be optimized still significantly.

\section{ASSOCIATED CONTENT}

\section{S Supporting Information}

The synthetic procedures for the composite dumbbells with a silica or titania/silica core are described in Figure S1 and Table S1. Average dimensions of each part of asymmetric dumbbells are shown in Table S2. Optical microscope images of silica particles, PMMA-coated silica particles, and spherical polystyrene particles are shown in Figure S2. Wide views of the asymmetric dumbbells incorporating titania under electric fields are shown in Figure S3. XRD patterns of titania spheres with and without the hydrothermal treatment are shown in Figure S4. This material is available free of charge via the Internet at http://pubs.acs.org. 


\section{AUTHOR INFORMATION}

\section{Corresponding Author}

*Tel: +81-22-795-7239. Fax: +81-795-7241. E-mail: konno@ mickey.che.tohoku.ac.jp.

\section{Notes}

The authors declare no competing financial interest.

\section{ACKNOWLEDGMENTS}

This research was partially supported by the Ministry of Education, Culture, Sports, Science and Technology (22651053, 23246134, and 23681020).

\section{REFERENCES}

(1) Ge, J.; Lee, H.; He, L.; Kim, J.; Lu, Z.; Kim, H.; Goebl, J.; Kwon, S.; Yin, Y. Magnetochromatic Microspheres: Rotating Photonic Crystals. J. Am. Chem. Soc. 2009, 131, 15687-15694.

(2) Hosein, I. D.; Lee, S. H.; Liddell, C. M. Dimer-Based ThreeDimensional Photonic Crystals. Adv. Funct. Mater. 2010, 20, 30853091.

(3) Wang, C.; Yin, H.; Dai, S.; Sun, S. A General Approach to Noble Metal-Metal Oxide Dumbbell Nanoparticles and Their Catalytic Application for CO Oxidation. Chem. Mater. 2010, 22, 3277-3282.

(4) Liu, Y. D.; Fang, F. F.; Choi, H. J. Core-Shell Structured Semiconducting PMMA/Polyaniline Snowman-like Anisotropic Microparticles and Their Electrorheology. Langmuir 2010, 26, 1284912854 .

(5) Hosein, I. D.; Liddell, C. M. Convectively Assembled Asymmetric Dimer-Based Colloidal Crystals. Langmuir 2007, 23, 10479-10485.

(6) Snezhko, A. Non-equilibrium Magnetic Colloidal Dispersions at Liquid-Air Interfaces: Dynamic Patterns, Magnetic Order and SelfAssembled Swimmers. J. Phys: Condens. Matter 2011, 23, 153101.

(7) Love, J. G.; Urbach, A. R.; Prentiss, M. G.; Whitesides, G. M. Three-Dimensional Self-Assembly of Metallic Rods with Submicron Diameters Using Magnetic Interactions. J. Am. Chem. Soc. 2003, 125, 12696-12697.

(8) Ding, T; Song, K.; Clays, K.; Tung, C. H. Fabrication of 3D Photonic Crystals of Ellipsoids: Convective Self-Assembly in Magnetic Field. Adv. Mater. 2009, 21, 1936-1940.

(9) Zerrouski, D.; Baudry, J.; Pine, D.; Chaikin, P.; Bibette, J. Chiral Colloidal Clusters. Nature 2008, 455, 380-382.

(10) Lee, S. H.; Liddell, C. M. Anisotropic Magnetic Colloids: A Strategy to Form Complex Structures Using Nonspherical Building Blocks. Small 2009, 5, 1957-1962.

(11) Herlihy, K. P.; Nunes, J.; DeSimone, J. M. Electrically Driven Alignment and Crystallization of Unique Anisotropic Polymer Particles. Langmuir 2008, 24, 8421-8426.

(12) Singh, J. P.; Lele, P. P.; Nettesheim, F.; Wanger, F. N.; Furst, E. M. One- and Two-Dimensional Assembly of Colloidal Ellipsoids in AC Electric Fields. Phys. Rev. E 2009, 79, 050401.

(13) Demirörs, A. F.; Johnson, P. M.; van Kats, C. M.; van Blaaderen, A.; Imhof, A. Directed Self-Assembly of Colloidal Dumbbells with an Electric Field. Langmuir 2010, 26, 14466-14471.

(14) Kuijk, A.; van Blaaderen, A.; Imhof, A. Synthesis of Monodisperse, Rodlike Silica Colloids with Tunable Aspect Ratio. J. Am. Chem. Soc. 2011, 13, 2346-2349.

(15) Kramer, H.; Deggelmann, M.; Graf, C.; Hagenbüchle, M.; Johner, C.; Weber, R. Electric Birefringence Measurements in Aqueous fd Virus Solutions. Macromolecules 1992, 25, 4325-4328.

(16) Mittal, M.; Furst, E. C. Electric Field-Directed Convective Assembly of Ellipsoidal Colloidal Particles To Create Optically and Mechanically Anisotropic Thin Films. Adv. Funct. Mater. 2009, 19, $3271-3278$

(17) Rotunno, M.; Bellini, T.; Lansac, Y.; Glaser, M. A. Phase Behavior of Polarizable Spherocylinders in External Fields. J. Chem. Phys. 2004, 121, 5541.

(18) Nagao, D.; Hashimoto, M.; Hayasaka, K; Konno, M. Synthesis of Anisotropic Polymer Particles with Soap-Free Emulsion Polymer- ization in the Presence of a Reactive Silane Coupling Agent. Macromol. Rapid Commun. 2008, 29, 1484-1488.

(19) Nagao, D.; van Kats, C. M.; Hayasaka, K.; Sugimoto, M.; Konno, M.; Imhof, A.; van Blaaderen, A. Synthesis of Hollow Asymmetrical Silica Dumbbells with a Movable Inner Core. Langmuir 2010, 26, 5208-5212.

(20) Mine, E.; Hirose, M.; Nagao, D.; Kobayashi, Y.; Konno, M. Synthesis of Submicrometer-Sized Titania Spherical Particles with a Sol-Gel Method and Their Application to Colloidal Photonic Crystals. J. Colloid Interface Sci. 2005, 291, 162-168.

(21) Leunissen, M. E.; Christova, C. G.; Hynninen, A.-P.; Royall, C. P.; Campbell, A. I.; Imhof, A.; Dijkstra, M. D.; van Roij, R.; van Blaaderen, A. Nature 2005, 437, 235-240.

(22) Li, J.; Seok, S. I.; Chu, B.; Dogan, F.; Zhang, Q.; Wang, Q. Nanocomposites of Ferroelectric Polymers with $\mathrm{TiO}_{2}$ Nanoparticles Exhibiting Significantly Enhanced Electrical Energy Density. Adv Mater. 2009, 21, 217-221.

(23) Karunagarana, B.; Chung, S. J.; Suh, E.-K.; Mangalaraj, D. Dielectric and Transport Properties of Magnetron Sputtered Titanium Dioxide Thin Films. Physica B 2005, 369, 129-134.

(24) Fontanella, J.; Johnston, R. L.; Sigel, G. H., Jr. The Dielectric Properties of As-Received and Gamma Irradiated Fused Silica. J. NonCryst. Solids 1979, 31, 401-414.

(25) Jo, M.-H.; Park, H.-H.; Kim, D.-J.; Hyun, S.-H.; Choi, S.-Y.; Paik, J.-T. $\mathrm{SiO}_{2}$ Aerogel Film as a Novel Intermetal Dielectric. J. Appl. Phys. 1997, 82, 1299-1304. 